\title{
Low-energy plasmons in quantum-well and surface states of metallic thin films
}

\author{
V. M. Silkin, ${ }^{1,2,3}$ T. Nagao, ${ }^{4}$ V. Despoja, ${ }^{2,5}$ J. P. Echeverry, ${ }^{2,5}$ S. V. Eremeev, ${ }^{2,6,7}$ E. V. Chulkov, ${ }^{1,2,5}$ and P. M. Echenique ${ }^{1,2,5}$ \\ ${ }^{1}$ Departamento de Física de Materiales, Facultad de Ciencias Químicas, Universidad del País Vasco, Apdo. 1072, \\ E-20080 San Sebastián/Donostia, Spain \\ ${ }^{2}$ Donostia International Physics Center (DIPC), P. de Manuel Lardizabal 4, E-20018 San Sebastián/Donostia, Spain \\ ${ }^{3}$ IKERBASQUE, Basque Foundation for Science, E-48011 Bilbao, Spain \\ ${ }^{4}$ International Center for Materials Nanoarchitectonics (MANA), National Institute for Materials Science (NIMS), Tsukuba 305-0044, Japan \\ ${ }^{5}$ Centro de Física de Materiales CFM, Materials Physics Center MPC, Centro Mixto CSIC-UPVIEHU, P. de Manuel Lardizabal 5, \\ E-20018 San Sebastián/Donostia, Spain \\ ${ }^{6}$ Institute of Strength Physics and Materials Science, 634021 Tomsk, Russia \\ ${ }^{7}$ Tomsk State University, 634050 Tomsk, Russia
}

(Received 28 January 2011; revised manuscript received 21 July 2011; published 10 October 2011)

\begin{abstract}
We studied low-energy plasmons in ultrathin films of silver in the thickness regimes where the surface states as well as quantum-well states must play significant roles. Realistic band structure was adopted for assessing the quantum-mechanical effect on the low-energy charge dynamics. In addition to the expected quasi-twodimensional plasmon mode, we find the modes that resemble an acoustic surface plasmon on semi-infinite metal surfaces and an additional plasmon mode related to the interband transitions between the two slab-split surface states. It is found that the dispersion of the latter mode is almost identical to the acoustic surface plasmon except the energy offset at small momenta values. The peaks in the surface response function related to interband transitions between the surfacelike states and bulklike states are identified as well. The present work elucidates the role of quantized electronic states and surface states on the plasmonic excitations in the ultimately thin films potentially used in the future nano-optics devices.
\end{abstract}

DOI: 10.1103/PhysRevB.84.165416

PACS number(s): 73.21.Fg, 73.20.At, 73.22.Lp, 78.66.Bz

\section{INTRODUCTION}

Electrodynamical response of thin metallic films is attracting growing interest in the areas of photonics and plasmonics research such as light-emitting devices, solar cells, and sensors. ${ }^{1-4}$ Very often noble metals such as Au and Ag are used, and the electromagnetic response of such nanometric objects, in particular the dominating collective electronic excitations, or plasmons, can be readily understood and described within classical electrodynamics to a certain extent. Examples include the Mie plasmon ${ }^{5-7}$ in nanoparticles and surface plasmons in thin films ${ }^{8-10}$ and metal surfaces. ${ }^{11-13}$

The conventional three-dimensional (3D) picture based on bulk electronic structure holds as long as the system size is far larger than the electron Fermi wavelength, $\lambda_{F}$. On the other hand, electrical and magnetic properties of a metallic object are affected markedly by size and shape when the size scales down to the level of $\lambda_{F}$. As most metals have a $\lambda_{F}$ comparable with interatomic distances, ${ }^{14}$ when the object size reduces to this scale, an effect similar to optical Fabry-Pérot interference comes into play. ${ }^{15-18}$ As a result, when characteristic size decreases to the atomic scale, quantum effects become more important and classical theories can be insufficient to describe excitations in such systems.

The effect of quantization of electronic states in the perpendicular direction on the excitation spectra of thin films was investigated in detail for the case of semiconducting heterostructures. In particular, it was shown that the sub-band [or quantum-well states (QWSs)] structure in these systems, arising from a finite thickness of a quasi-2D electron gas, gives rise to quantum effects that are absent in a purely $2 \mathrm{D}$ electron gas. ${ }^{19,20}$ Thus, in addition to a low-energy intra-subband plasmon, the inter-sub-band transitions corresponding to charge-density oscillations perpendicular to the film plane lead to the appearance of a high-energy collective mode called the inter-sub-band plasmon. ${ }^{21-23}$ The properties of such modes ${ }^{24,25}$ and their modification and interaction due to variations in a number of QWS bands were studied as well. ${ }^{23,26}$

In the early theoretical works on the quantum-mechanical electrodynamical response of metal surfaces as well as in the electronic band structure calculations, the jellium model ${ }^{27}$ has been widely used to gain basic insight into the nature of electronic excitations. However, the model has some drawbacks that limit its application to real metal surfaces. One major disadvantage is that it fails to reproduce the surface electronic states existing on real metal surfaces. ${ }^{28-30}$ These states do not exist in the bulk and their wave function is localized at the surface. These states must play an important role in electrical as well as in optical properties of ultrathin films. In particular, recently it was found that the $s-p_{z}$ surface states characterized by a partly occupied parabolic-like dispersion, even hosting a small amount of the total electronic charge at the surface, can dramatically affect the low-energy dynamical properties of real metal surfaces like noble metal surfaces. ${ }^{31,32}$ In addition to the well-known symmetric mode of the surface plasmons in thin films (a $\omega_{\mathrm{sp}}^{-}$mode), ${ }^{33}$ these surface states support new type of low-energy plasmonics excitation named the acoustic surface plasmon. ${ }^{31}$

In the present study we concentrate on the low-energy part of the excitation spectra, especially on the two quantummechanically screened two-dimensional- (2D) like plasmons. They are related to the presence of the slab-split surface-state bands and evolve into an acoustic surface plasmon ${ }^{31}$ on the metal surfaces. Moreover, some other features in the excitation spectra, which are missing in the jellium model, due to the 
interband transitions between the bulklike and surfacelike electronic states, are found. We believe the result presented here provides useful knowledge for the future nanophotonic devices when they scale down to the thickness regime where the quantization of the electronic state and the contribution of the surface electronic states become significant.

The rest of the paper is organized as follows. A short outline of the models used and the calculation method is given in Sec. II. In Sec. III we present and discuss in detail the calculation results. Finally, the conclusions are drawn in Sec. IV. Atomic units are used $\left(e^{2}=\hbar=m_{e}=1\right)$ unless otherwise stated.

\section{CALCULATION METHOD}

When a perturbing electric charge is located far from one side of the film the differential cross section for its scattering with energy $\omega$ and momentum $\operatorname{transfer}^{34} q=|\mathbf{q}|$ is proportional to the imaginary part of a surface response function $g(q, \omega)$ defined as ${ }^{35}$

$$
g(q, \omega)=-\frac{2 \pi}{q} \int d z \int d z^{\prime} \chi\left(z, z^{\prime}, q, \omega\right) e^{q\left(z+z^{\prime}\right)},
$$

which depends on the film electronic properties only. This quantity is relevant in the description of surface collective excitations measured in electron energy-loss experiments. ${ }^{12}$ Here $\chi\left(z, z^{\prime}, q, \omega\right)$ is the density response function of an interacting electron system that determines, within linear response theory, the electron density $n^{\text {ind }}(z, q, \omega)$ induced in the system by an external potential $V^{\text {ext }}\left(z^{\prime}, q, \omega\right)$ according to

$$
n^{\text {ind }}(z, q, \omega)=\int d z^{\prime} \chi\left(z, z^{\prime}, q, \omega\right) V^{\text {ext }}\left(z^{\prime}, q, \omega\right) .
$$

The collective electronic excitations in thin films then can be traced to the peaks in surface loss function defined as an imaginary part of $g, \operatorname{Im}[g(q, \omega)]$.

In the framework of time-dependent density functional theory, $\chi$ is the solution of an integral equation

$$
\begin{aligned}
\chi\left(z, z^{\prime}, q, \omega\right)= & \chi^{o}\left(z, z^{\prime}, q, \omega\right)+\int d z_{1} \int d z_{2} \chi^{o}\left(z, z_{1}, q, \omega\right) \\
& \times\left[v_{c}\left(z_{1}, z_{2}, q\right)+K_{x c}\left(z_{1}, z_{2}, q, \omega\right)\right] \chi\left(z_{2}, z^{\prime}, q, \omega\right),
\end{aligned}
$$

with $\chi^{o}$ being the response function of a noninteracting electron system. In Eq. (3) $v_{c}\left(z, z^{\prime}, q\right)=-\frac{2 \pi}{q} e^{q\left|z-z^{\prime}\right|}$ stands for the 2D Fourier transform of the bare Coulomb potential and $K_{x c}$ accounts for the exchange-correlation effects. In the present work, we use a random-phase approximation when $K_{x c}$ is set to zero, i.e., the dynamical exchange-correlation effects are ignored. In the previous work on semiconductor heterostructures $^{25}$ it was shown that the inclusion of the exchange-correlation kernel may induce a shift of the intersub-band plasmon energy by around $10 \%$ and produce an additional broadening. These are the cases for a larger density parameter (or smaller electron kinetic energy) that are typical for QWS by semiconductor heterostructures. For the case of metallic systems, the density parameters are smaller (larger electron kinetic energy) and should be smaller the exchange correlation effects. The previous studies of collective excitations at the surfaces ${ }^{9,20,36-38}$ and in the bulk ${ }^{39,40}$ of many "metallic" systems suggest that the effect should have a smaller impact for the systems studied here.

The evaluation of $\chi^{o}\left(z, z^{\prime}, q, \omega\right)$ for a slab consisting of $n$ monolayers (MLs) according to

$$
\begin{aligned}
\chi^{o}\left(z, z^{\prime}, q, \omega\right)= & \frac{2}{S} \sum_{l, l^{\prime}} \phi_{n l}(z) \phi_{n l^{\prime}}^{*}(z) \phi_{n l^{\prime}}\left(z^{\prime}\right) \phi_{n l}^{*}\left(z^{\prime}\right) \\
& \times \sum_{\mathbf{k}} \frac{f_{\mathbf{k}}^{n l}-f_{\mathbf{k}+\mathbf{q}}^{n l^{\prime}}}{E_{\mathbf{k}}^{n l}-E_{\mathbf{k}+\mathbf{q}}^{n l^{\prime}}+\omega+i \eta}
\end{aligned}
$$

is performed in a reciprocal space using an approach developed by Eguiluz. ${ }^{41,42}$ In Eq. (4) a factor 2 accounts for spin, $S$ is the normalization area, and $\eta$ is a positive infinitesimal. In the present calculations we take $\eta=1 \mathrm{meV}$. At $T=0$ the Fermi factor $f_{\mathbf{k}}^{n l}$ is the Heaviside step function. The sums over $l$ and $l^{\prime}$ include both occupied and unoccupied electronic states. Every energy band $E_{\mathbf{k}}^{n l}$ has a parabolic dispersion

$$
E_{\mathbf{k}}^{n l}=E^{n l}+\frac{\mathbf{k}^{2}}{2 m_{n l}^{*}},
$$

where the one-particle eigenenergies $E^{n l}$ and wave functions $\phi_{n l}(z)$ are solutions of the one-dimensional Schrödinger equation

$$
\left[-\frac{1}{2} \frac{d^{2}}{d z^{2}}+V(z)\right] \phi_{n l}(z)=E^{n l} \phi_{n l}(z) .
$$

In Eq. (5) the value of an effective mass $m_{n l}^{*}$ in each band can differ from unity in order to reproduce the realistic $s-p$ surface electronic structure of $\operatorname{Ag}(111)$. In this case, evaluation of $\chi^{o}$ via Eq. (4) is performed as described in Ref. 32. Present calculations were made for the Ag slabs consisting of 1-10 MLs. For comparison, similar calculations are performed for slabs consisting of 17 and 31 layers. For the description of the Ag electronic structure we employed two models for the effective one-electron $V(z)$ potential in Eq. (6). The first is a conventional self-consistent jellium model. ${ }^{27}$ In a second model the $\operatorname{Ag}(111)$ electronic structure at $k=0$ is described with the use of a model potential. ${ }^{43,44}$ In the previous works $^{31,32,45,46}$ the adequacy of this model potential has been demonstrated for the description of collective electronic excitations at metal surfaces. In the case of the use of the model potential we obtain two sets of data. In the first one, the effective masses of the slab quantum states are set to unity and, in the second one, they differ from unity in order to reproduce the realistic $s$ - $p$ band structure of the $\operatorname{Ag}(111)$ surface. In the following, we refer to them as the $m=1 \mathrm{Ag}(111)$ and $m \neq 1 \mathrm{Ag}(111)$ models, respectively. In the $m \neq 1 \mathrm{Ag}(111)$ model for the quantum states, with energies falling in the energy range for the projected Ag bulk states, ${ }^{44}$ the effective masses are gradually changed from unity at the bottom of the valence band to 0.25 at its upper border, whereas masses for two states located in the bulk energy gap and evolving into the $\operatorname{Ag}(111)$ surface state with the increasing slab thickness are set to 0.44 . For example, the electronic structure for the 3-ML slab in these three models is presented in Fig. 1. The electronic structure for the 31-ML slab that reproduces well the situation for a semi-infinite system is shown in Fig. 2. Figure 3 presents the one-particle energies at $k=0$ calculated 


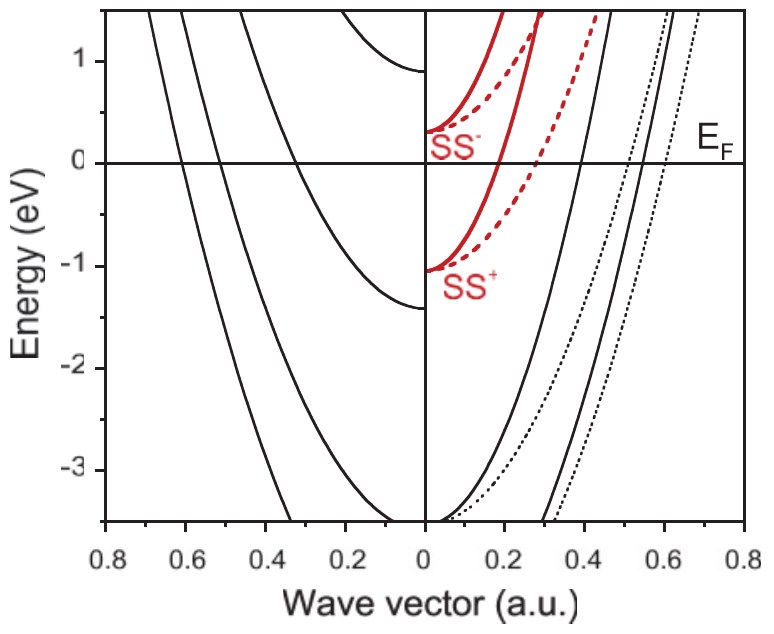

FIG. 1. (Color online) (Right) Calculated band structure for the 3-ML $\operatorname{Ag}(111)$ slab. The energy positions at $k=0$ are obtained with the use of the $\operatorname{Ag}(111)$ model potential. ${ }^{44}$ Dispersion of the slab energy bands with effective masses set to unity (dashed lines) and realistic ones (solid lines) is shown. A pair of even and odd energy bands evolving into the $\operatorname{Ag}(111)$ surface state on increase of the slab thickness for realistic (unity) effective masses is presented by thick solid (dashed) lines and denoted as $\mathrm{SS}^{+}$and $\mathrm{SS}^{-}$, respectively. (Left) Calculated band structure for the jellium slab with thickness equal to three Ag MLs. All energies are relative to the Fermi level, $E_{F}$.

with the use of a model potential ${ }^{44}$ for slabs of different thickness in comparison with available photoemission data obtained in thin $\operatorname{Ag}(111)$ films grown on a silicon substrate. ${ }^{47}$ One can note that the agreement with experimental data for the bulklike electronic states is rather good. Some existing differences are explained by the hybridization of the silver QWSs with the Si electronic states. ${ }^{48}$ Nevertheless, in the calculated energy spectrum for thicker slabs, the number of states and the energy splitting between states is nearly the same as the experimentally observed ones. Moreover, the energy of the slab $\mathrm{SS}^{+}$and $\mathrm{SS}^{-}$states, analogs of a $\mathrm{Ag}(111)$ surface state, is nicely reproduced by the calculation although most probably only a single lower-energy SS state is realized in the existing experiments due the substrate influence.

In Figs. 1 and 2 it is clearly seen that the band structure of the $\mathrm{Ag}(111)$ films evaluated in the jellium model and with use of a realistic one-particle model potential differ rather substantially in the vicinity of the Fermi level. The differences are also seen in the charge-density distribution of the corresponding QWSs evaluated in both the models. For example, the calculated results for the 8-ML slab are shown in Fig. 4. In this figure it is seen that in the jellium calculation the peaks in the charge density of all the QWSs have almost the same amplitude in the entire thickness of the film. However, in the $\operatorname{Ag}(111)$ case, the presence of the modulation in the potential ${ }^{44}$ inside the slab produces notable modifications in the charge-density distribution of all the QWSs. Even in the lowest-energy QWS some extra modulation can be seen in Fig. 4(a). This effect is getting stronger with increasing number of the QWS. Thus, the charge density of the QWS labeled by 7 is mainly localized in the center of the slab with a strong reduction of its weight in the subsurface regions. In contrast, the two highest QWSs, labeled as $\mathrm{SS}^{-}$and $\mathrm{SS}^{+}$, have strong localization at the surfaces and

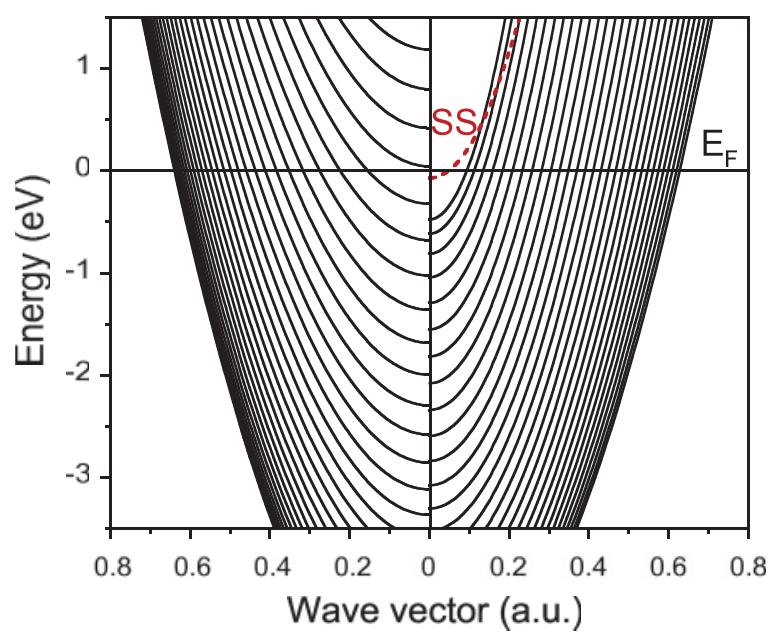

FIG. 2. (Color online) (Right) Calculated band structure for the 31-ML $\operatorname{Ag}(111)$ slab. The energy positions at $k=0$ are obtained with the use of the $\operatorname{Ag}(111)$ model potential. ${ }^{44}$ Dispersion of the slab energy bands with realistic effective masses is shown. Note that for this slab thickness the splitting of the even $\mathrm{SS}^{+}$and odd $\mathrm{SS}^{-}$ surface states is negligible and only a doubly degenerate surface state SS exists. (Left) Calculated band structure for the jellium slab with thickness equal to $31 \mathrm{Ag}$ MLs. Note that, in this case, absence of an energy gap in the projected bulk electronic structure does not allow appearance of any surface state. All energies are relative to the Fermi level, $E_{F}$.

on thickness increase they evolve into the conventional surface state of the $\operatorname{Ag}(111)$ surface.

\section{CALCULATION RESULTS AND DISCUSSION}

We start by showing the calculated results for the surface loss function $\operatorname{Im}[g(q, \omega)]$, a quantity directly related to the

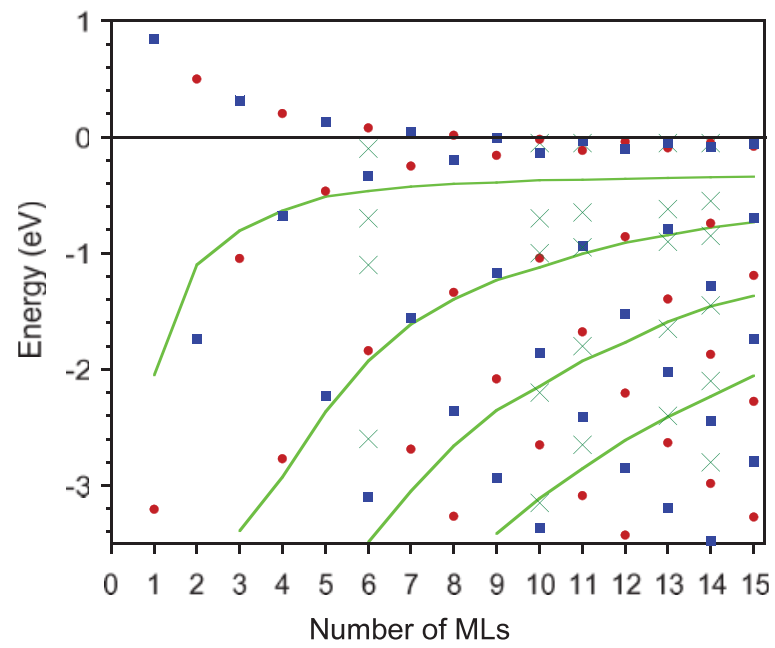

FIG. 3. (Color online) Energy values at the $k=0$ point for quantum-well states in the $\operatorname{Ag}(111)$ slabs versus number $n$ of MLs obtained with the use of a model potential of Ref. 44. Dots and squares stand for even and odd quantum states, respectively. Crosses show the experimental values measured in Ag overlayers on $\mathrm{Si}(111) .{ }^{47}$ Solid lines show energies calculated in Ref. 47 according to the phase-shift quantization rule. 


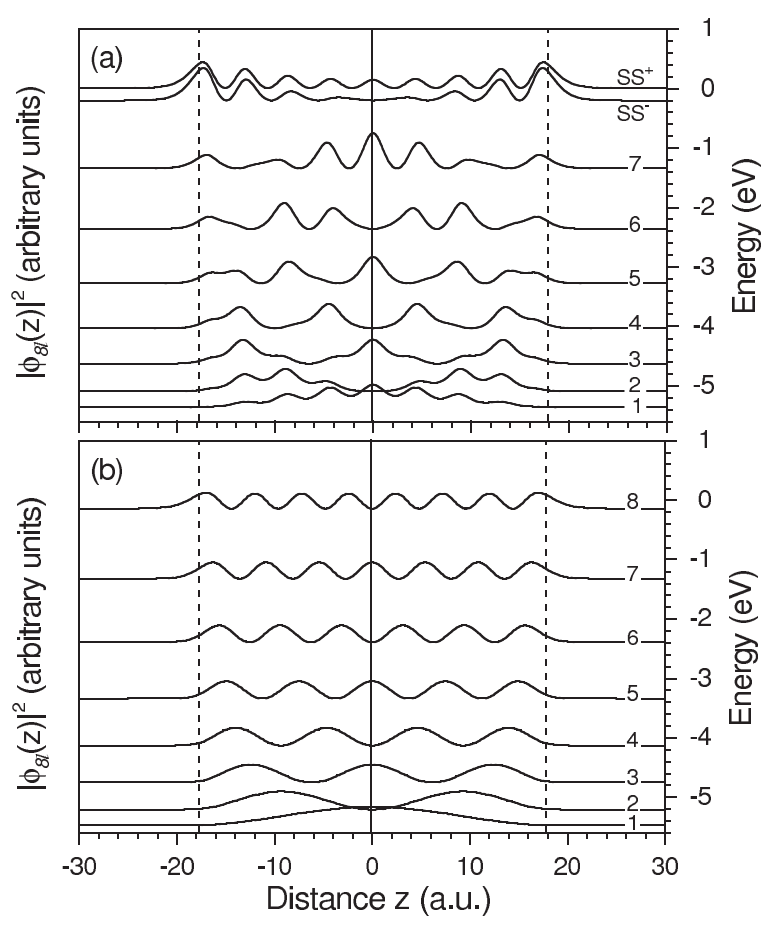

FIG. 4. Charge-density distribution of the lowest-energy QWSs, $\left|\phi_{n l}(z)\right|^{2}$, as a function of $z$ in the 8-ML slab: (a) the $\mathrm{Ag}(111)$ model potential ${ }^{44}$ and (b) the jellium calculations. The zero position for each curve is located at the corresponding energy $E^{8 l}$ shown on the right. The zero energy is at the Fermi level. The QWS number is counted from the bottom. In the $\operatorname{Ag}(111)$ case the states corresponding to the surfacelike states are denoted by $\mathrm{SS}^{-}$and $\mathrm{SS}^{+}$according to their symmetry respective to the $z=0$ plane.

spectra measured in angle-resolved surface energy-loss experiments for the $\operatorname{Ag}(111)$ films consisting of 1-10 MLs. The data for the $m \neq 1 \mathrm{Ag}(111)$ slabs are shown in Fig. 5. For comparison, in the same figure, the data for the 17-ML and 31-ML slabs are shown as well. Comparison of the calculated $\operatorname{Im}[g(q, \omega)]$ for the $m=1 \operatorname{Ag}(111)$ and $m \neq 1 \operatorname{Ag}(111)$ models demonstrates that they are qualitatively similar. The main differences among them are observed in energetic positions of essentially the same features. These differences are explained by the different energy dispersions of the partly occupied energy bands employed in these two sets of the calculations. Due to the qualitative similarity of these two figures, in the following we shall mainly refer to the $m \neq 1 \operatorname{Ag}(111)$ case, although some differences among them will be addressed as well.

The most prominent feature in the surface loss function of Fig. 5 is a strongly dispersing peak corresponding to the conventional $\omega_{\mathrm{sp}}^{-}$mode presented in all the spectra for the thin films. The same mode is also observed in the surface excitation spectra obtained in the jellium model shown in Fig. 6 for the slabs of the same thicknesses. Dispersion of this mode in all the models is very similar and can be described reasonably by expression for the lower-energy $\omega_{\mathrm{sp}}^{-}$ plasmon mode in a thin film. ${ }^{33}$ One can note in Figs. 5 and 6 that this mode is the only visible feature in the case of the 1-ML slab. A similar mode was observed in a metallic ML adsorbed on silicon. ${ }^{20,49-54}$ The properties of this mode as well as its transformation to the conventional surface plasmon on increasing the film thickness and to localized plasmon in confined structures were investigated in preceding experimental $^{20,49,53-58}$ and theoretical studies. ${ }^{59-67}$

One can see in Fig. 6 that surface excitation spectra evaluated in the jellium model are rather featureless in this low-energy domain compared to Fig. 5. This is in agreement with other jellium-like calculations. ${ }^{59}$ Nevertheless, in thin films, in addition to a dominant strongly dispersing peak $\omega_{\mathrm{sp}}^{-}$, in the "small $q$-small $\omega$ " phase-space region one can detect weak features having their origin in intraband transitions in the QWSs (which have a 2D-like character in these relatively thin slabs). Also, some faint signals due to interband transitions can be noted. However, the effect of these transitions on surface excitation spectrum is very small in comparison with the strength of the features observed in Fig. 5 for the $\operatorname{Ag}(111)$ films with the realistic band structure.

In the excitation spectra presented in Fig. 5, in addition to the $\omega_{\mathrm{sp}}^{-}$peak, many other features related to the intraand interband transitions can be seen. The most prominent ones among them are discussed below. In the lower-energy region one can detect a rather sharp peak corresponding to the acoustic surface plasmon (ASP) with a soundlike dispersion for all films with number of MLs greater than one, i.e., starting at the $(q=0, \omega=0)$ point and proceeding almost linearly with momentum at small $q$. In order to elucidate the origin of this mode, one should refer to the band structure of the films. As follows from Fig. 3, starting from $n=2$, in all the films more than one energy band is partly occupied. Hence, at the Fermi level, the carriers in several energy bands can be excited. For the partly occupied energy bands with the parabolic dispersion of Eq. (5), the Fermi velocities defined as $v_{F}^{n l}=\sqrt{2\left(E_{F}-E_{F}^{n l}\right) / m_{n l}^{*}}$ (where $n$ is the number of MLs in a film and $l$ is the number of the quantum state) differ markedly because of very different binding energies, $E_{F}^{n l}$. Consequently, there are conditions for the existence of a so-called acoustic plasmon ${ }^{68-70}$ or its modification in the case of metal surfaces: an acoustic surface plasmon. ${ }^{31}$

The dispersion of this mode follows closely the upper border of the intraband electron-hole continuum corresponding to the energy band with the slowest carriers at the Fermi surface. ${ }^{31,71}$ Very recently, this kind of plasmon has been observed experimentally on a variety of metal surfaces. ${ }^{45,72,73}$ One can image the ASP as a result of the screening of carriers in the energy band with slowest Fermi velocity by the carriers in the other bands with larger Fermi velocities. This can be observed in Fig. 7 where we present the induced charge density $n^{\text {ind }}(z, q, \omega)$ produced by the external potential

$$
V^{\mathrm{ext}}(z, q, \omega)=-\frac{2 \pi}{q} e^{q z} e^{-i \omega t}
$$

for the 8-ML $m \neq 1 \mathrm{Ag}(111)$ film calculated at $q=0.02$ a.u.. One can see how, at $\omega=0.1 \mathrm{eV}$, along the $z$ direction, the regions of positive charge in $\operatorname{Im}\left[n^{\text {ind }}(z, q, \omega)\right]$ correspond closely to the peaks of the charge density of the $\mathrm{SS}^{-}$quantum state (presented in Fig. 4), whereas the regions of negative $\operatorname{Im}\left[n^{\text {ind }}(z, q, \omega)\right]$ reflect the screening of these regions by the carriers in the other QWSs. The same is observed in the $\operatorname{Re}\left[n^{\text {ind }}(z, q, \omega)\right]$, although at the upper surface of the slab 

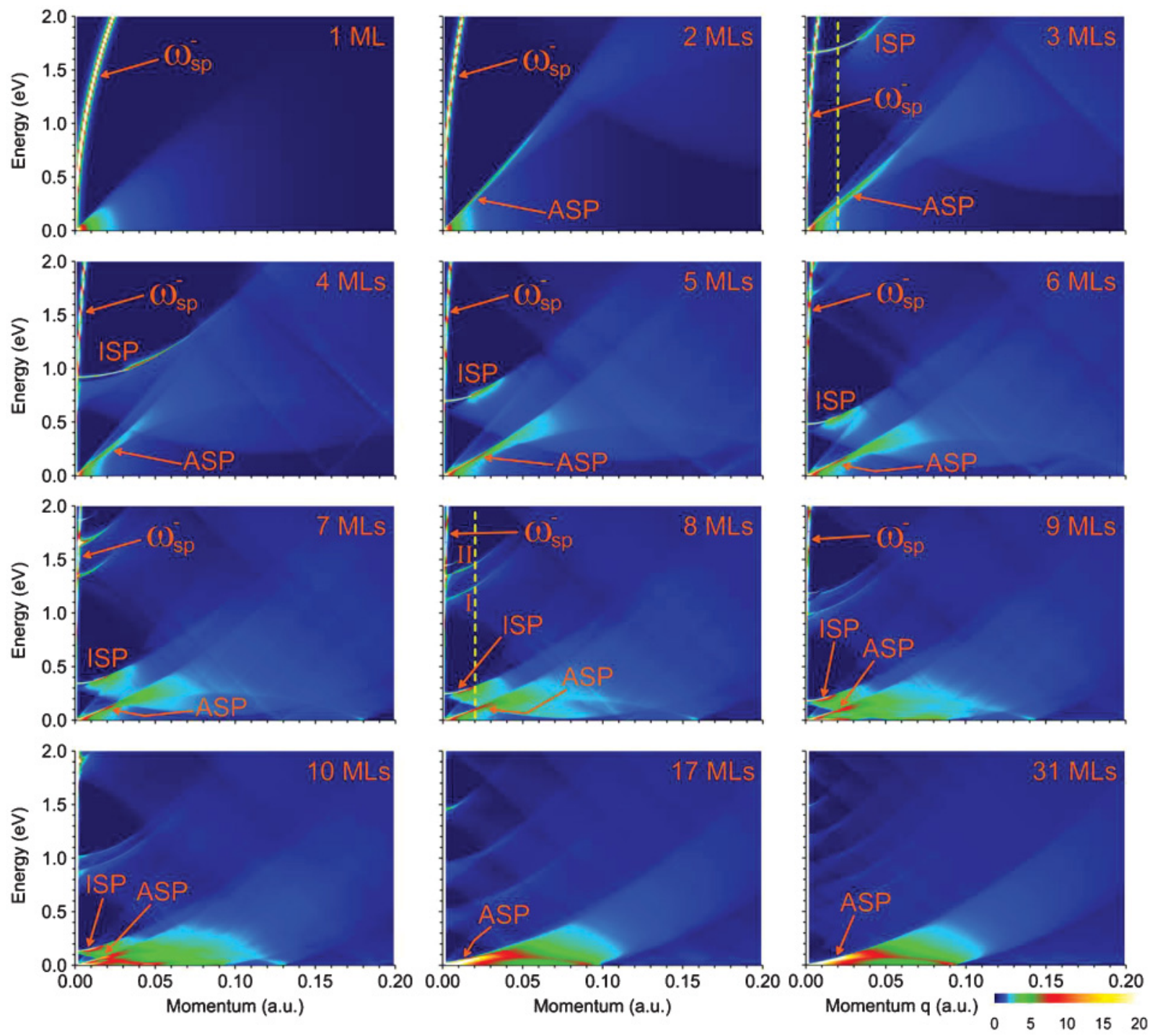

FIG. 5. (Color) Normalized surface loss function $\operatorname{Im}[g(q, \omega)] / q \omega$ for the 1- to 10-, 17-, and 31-ML $\operatorname{Ag}(111)$ films evaluated with the use of realistic effective masses in energy band dispersions. The strongly dispersing peak corresponding to a conventional $\omega_{\mathrm{sp}}^{-}$mode of a thin film is denoted by the corresponding symbol. Acoustic surface plasmon dispersion is labeled with "ASP." Peaks having their origin in the interband transitions between the energy-split $\mathrm{SS}^{+}$and $\mathrm{SS}^{-}$quantum states are denoted with "ISP". The dashed vertical lines for the 3-ML and 8-ML films correspond to cuts along which the induced charge density and the surface loss function are presented in Figs. 7, 8, and 9.

the picture is obscured by a strong positive peak due to a quasistatic extra screening charge. In Fig. 8 we compare the induced charge density corresponding to the ASP calculated at $q=0.02$ a.u. for the $m \neq 1 \mathrm{Ag}(111)$ 3-ML and 8-ML films. In the case of the 3-ML film one can see that $\operatorname{Im}\left[n^{\text {ind }}(z, q, \omega)\right]$ at $\omega=0.25 \mathrm{eV}$, corresponding to the ASP energy at this $q$, has nearly the same amplitude in all atomic planes, whereas in the 8-ML film the ASP-induced charge density is mainly confined to the surface and has a significantly smaller amplitude inside the film.

From Figs. 5 and 6 it is clear that the strength of the ASP peak is significantly weaker than the signal from the conventional $\omega_{\mathrm{sp}}^{-}$plasmon. However, in the low-energy domain, the ASP mode is the only dominant feature at finite momenta. This is demonstrated in Fig. 9, where we present, as an example, the surface loss function evaluated for 3-ML and
8-ML films at $q=0.02$ a.u., i.e., along the cuts shown by the vertical dashed lines in Figs. 5 and 6.

We now turn our discussion to the evolution of the ASP peak with the film thickness. As pointed out above, the slope of the ASP dispersion in each film is determined by the partly occupied energy band with the lowest Fermi velocity. For example, this band is shown by the lower thick dashed line $\mathrm{SS}^{+}$in Fig. 1 for the 3-ML $\mathrm{Ag}(111)$ film. Actually, this band is a symmetrical QWS of a film and, together with an antisymmetrical $\left(\mathrm{SS}^{-}\right)$state, is an analog of the $s-p_{z}$ surface state at the $\operatorname{Ag}(111)$ surface. As the number of atomic layers in the $\mathrm{Ag}(111)$ films increases, the energy splitting between the $\mathrm{SS}^{+}$and $\mathrm{SS}^{-}$bands decreases as can be observed in Fig. 3 . This is accompanied by the reduction of the binding energy of the lower-energy band in this pair. In turn, it leads to the lowering of the relevant Fermi velocity. As a result, the slope 

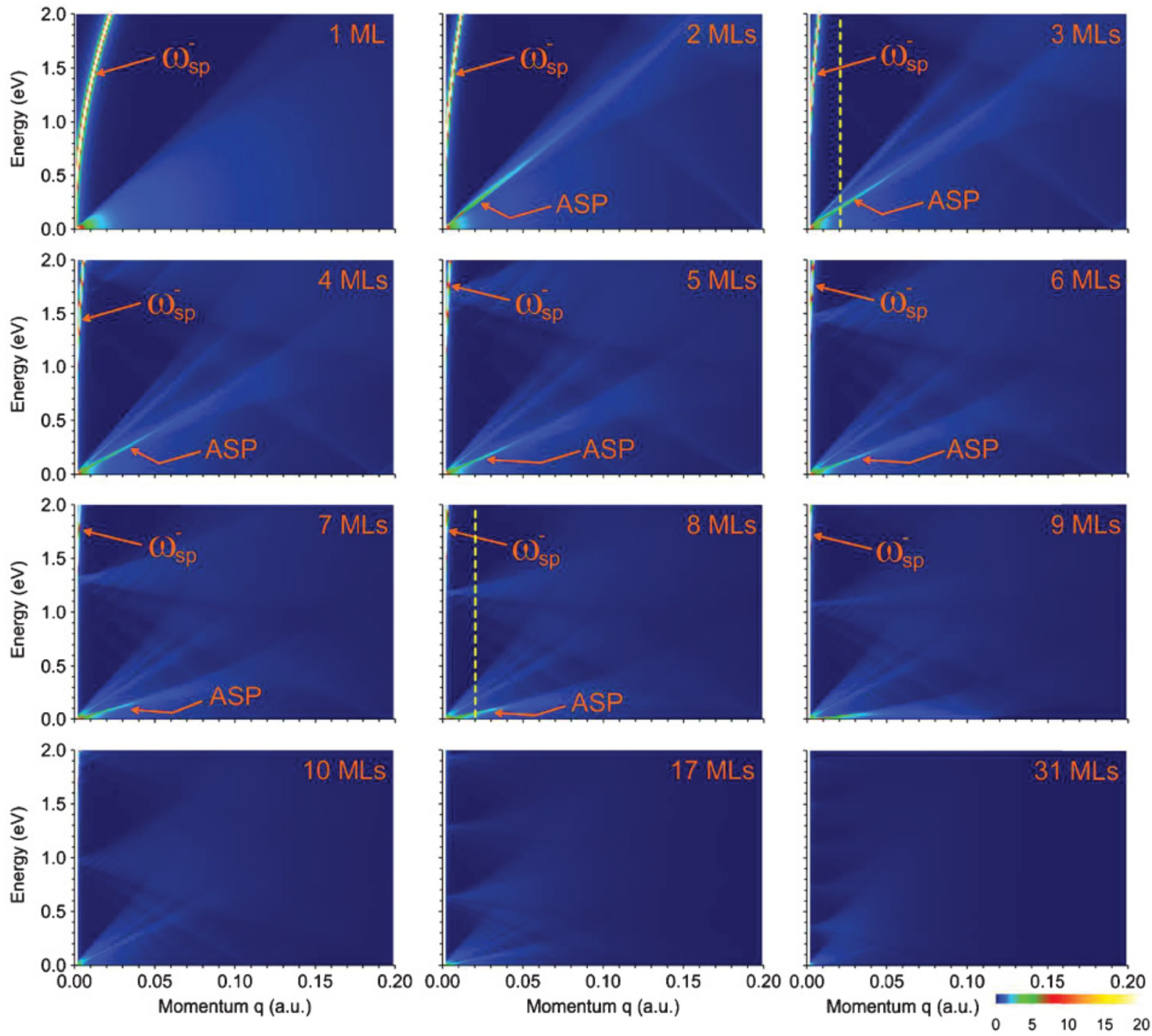

FIG. 6. (Color) Normalized surface-loss function $\operatorname{Im}[g(q, \omega)] / q \omega$ for the 1- to 10-, 17-, and 31-ML films calculated within the jellium model with the use of charge-density parameter $r_{s}=3$ a.u.. Strongly dispersing peak corresponding to a conventional $\omega_{\mathrm{sp}}^{-}$mode of a thin film is denoted by the corresponding symbol. Acoustic surface plasmon dispersion produced by a quantum-well state with slowest Fermi velocity (i.e., that with the smallest binding energy at $k=0$ point) is highlighted by the symbol "ASP". The dashed vertical lines for the 3-ML and 8-ML slabs correspond to cuts along which the surface loss function is presented in Fig. 9.

of the ASP dispersion in Fig. 5 is reduced as the slab thickness increases. At the same time, the energy position of the upper energy band is shifted down. As a consequence, for a certain film thickness (around $8 \mathrm{MLs}$ in the present case) the upper surface-state-like band starts to be occupied at $k=0$ as well. This can produce additional fine structures in the surface loss function at small energies and small momenta that cannot be resolved on the scale of Fig. 5.

Note that an acoustic-like ASP mode can be observed in surface excitation spectra for thin films, even in the jellium model calculations in Fig. 6. In this case, a role of an energy band with the slowest Fermi velocity is played by a partly occupied QWS with the smallest binding energy at $k=0$. The strongest ASP peak is seen for the 2-ML film with only two partly occupied energy bands. For thicker films, one can observe in Fig. 6 that the appearance of an additional state below the Fermi level at $k=0$ produces an additional peak in the surface loss function. This was also observed in Ref. 59. Nevertheless, in each case the strongest ASP-like peak in $\operatorname{Im}[g(q, \omega)]$ is produced due to the intraband transitions within the energy band with the slowest Fermi velocity, i.e., that with the smallest binding energy at $k=0$. However, the strength of the ASP peaks in the jellium calculations is significantly smaller in comparison with the ones obtained for the realistic Ag(111) films. An example of this can be seen in Fig. 9 for 3-ML and 8-ML films. The reduced strength of the ASP peak in the jellium model can be explained by the different nature of a relevant quantum state: in the jellium model it is an original QWS of a film characterized by a wave function that gradually reduces its weight at the slab surfaces on increasing thickness. Therefore, any signature of the ASP in the excitation spectrum of films vanishes very rapidly in the jellium calculations on increasing the number of MLs, as seen in Fig. 6. On the contrary, in the realistic $\mathrm{Ag}(111)$ film calculations, the relevant 


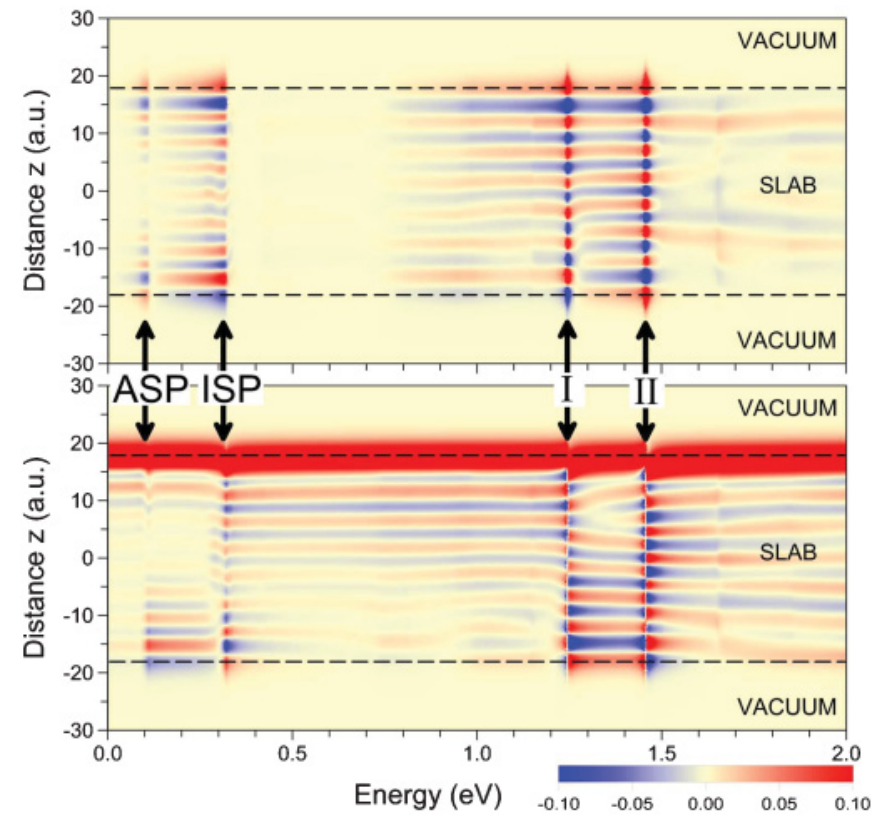

FIG. 7. (Color online) Two-dimensional plot of imaginary (top) and real (bottom) parts of induced charge density $n^{\text {ind }}(z, q, \omega)$ produced by the external potential of Eq. (7) versus energy $\omega$ and distance $z$. Calculations are preformed for the 8-ML $m \neq 1 \operatorname{Ag}(111)$ film at $q=0.02$ a.u.. The energy positions corresponding to the ASP and the ISP and the I and II modes are shown with arrows. The $\operatorname{Ag}(111)$ slab is delimited by two horizontal dashed lines.

quantum state is the analog of the $\operatorname{Ag}(111)$ surface state with wave function strongly localized at the film surfaces. Starting from a certain minimal film thickness, the localization of the wave function of this surface state is not affected by the film thickness. Consequently, the excitation spectrum of Fig. 5 is stabilized in the low-energy part, at the same time differing

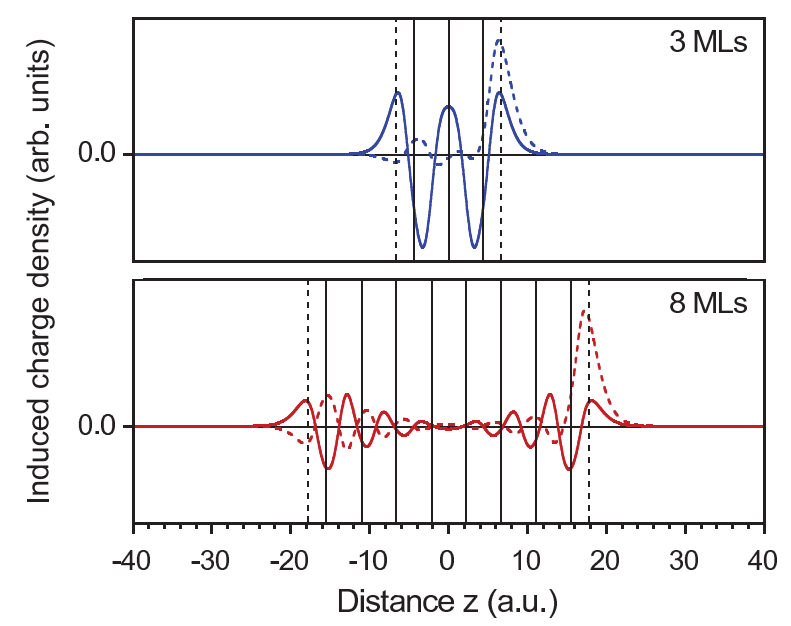

FIG. 8. (Color online) Imaginary (solid lines) and real (dashed lines) parts of the induced charge density, $n^{\text {ind }}\left(z, q, \omega_{\mathrm{ASP}}\right)$, corresponding to the ASP in the 3-ML (top panel) and 8-ML (bottom panel) $m \neq 1 \mathrm{Ag}(111)$ films. Calculations are for $q=0.02$ a.u. and $\omega_{\mathrm{ASP}}=0.25 \mathrm{eV}$ and $\omega_{\mathrm{ASP}}=0.1 \mathrm{eV}$ for the 3-ML and 8-ML films, respectively. Vertical solid lines show the atomic plane positions and vertical dashed lines delimit the slabs.

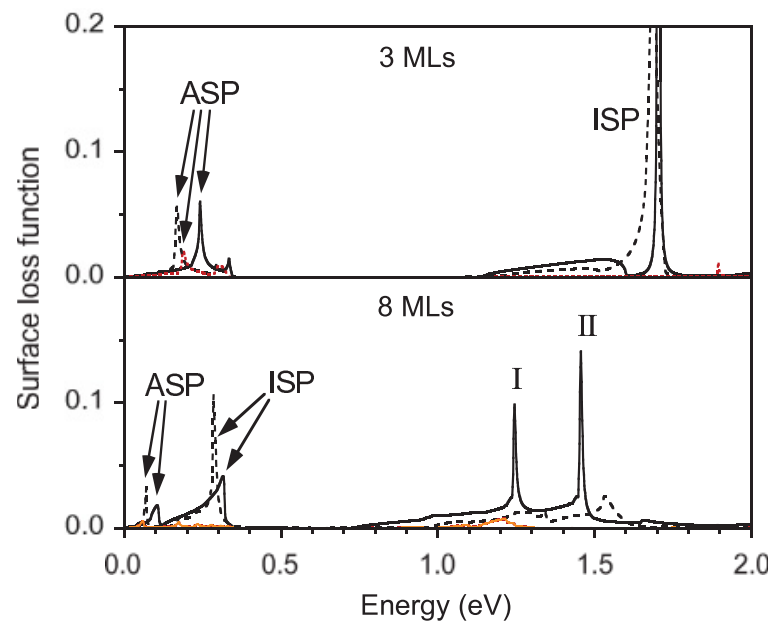

FIG. 9. (Color online) Surface loss function for the 3-ML (top panel) and 8-ML (bottom panel) films evaluated at $q=0.02$ a.u., i.e., along the cuts shown by the vertical dashed lines in Figs. 5 and 6. Solid lines show results obtained for the $m \neq 1 \operatorname{Ag}(111)$ model, whereas dashed lines correspond to the $m=1 \mathrm{Ag}(111)$ one. Dotted lines stand for the jellium calculations. The peaks corresponding to the ASP and the ISP and the I and II modes (explained in the text) are highlighted by the corresponding symbols.

from that obtained in the jellium model. We stress the fact that as the dispersion of the ASP is almost entirely determined ${ }^{71}$ by the Fermi velocity of the relevant energy band, the presence of the insulating substrate might modify its dispersion mostly via modifications in the film electronic structure. This is in contrast to the situation with conventional $\omega_{\mathrm{sp}}^{-}$plasmon whose dispersion is strongly affected by the dielectric function of the substrate.

In Figs. 5 and 9 one can observe other dispersing prominent peak denoted as "ISP" below $2 \mathrm{eV}$ in each film starting from $n=3$. In contrast to the ASP mode, its energy has finite value $\omega_{\min }^{n}$ at $q=0$. With increasing $n$ the energy of this peak is lowered and eventually this mode merges with the ASP one at large $n$. Thus, at $n=17$ and $n=31$ we obtain only a single ASP peak in the excitation spectrum. The threshold energy $\omega_{\min }^{n}$ for the ISP peak correlates with the energy splitting between the $\mathrm{SS}^{+}$and $\mathrm{SS}^{-}$quantum states. From this observation and analysis of the corresponding induced charge density we attribute this mode to the interband transitions between energy-split $\mathrm{SS}^{+}$and $\mathrm{SS}^{-}$electronic states. In the induced charge density corresponding to the ISP mode in Fig. 7 for the 8-ML film one can see that this mode has different symmetry respective to the ASP mode. Moreover, in thin films where the ASP and ISP modes have different energies their excitation is produced at both sides of the films. However, in thick films, where both modes are degenerate in energy, they are excited simultaneously. As a result, the corresponding charge can oscillate only at the surface exposed to external perturbation. As the jellium model does not allow the appearance of surface electronic states similar to the $\mathrm{SS}^{+}$ and $\mathrm{SS}^{-}$QWS states, in the corresponding surface excitation spectra of Fig. 6 a signature of a similar mode does not appear.

We now focus on two additional dispersing peaks in surface loss function seen in the $\operatorname{Ag}(111)$ calculations of Fig. 5. These 
peaks appear below $2 \mathrm{eV}$ in the surface excitation spectrum of the 7-ML films and their energy is down-shifted with the increase of a number of MLs in the film. As an example, these peaks, labeled with "I" and "II", are shown for the 8-ML film. Moreover, the energy distance between these two peaks reduces in concert. These peaks can also be seen in Fig. 9, presenting $\operatorname{Im}[g(q, \omega)]$ of the 8-ML $m \neq 1 \operatorname{Ag}(111)$ film evaluated at $q=0.02$ a.u., where the corresponding peaks are clearly visible at $\omega=1.25 \mathrm{eV}$ and $\omega=1.45 \mathrm{eV}$. Inspection of Fig. 5 reveals that the energy gap between these two peaks correlates with the energy splitting between $\mathrm{SS}^{+}$and $\mathrm{SS}^{-}$ states. We relate the origin of these two modes in the $m \neq 1$ $\mathrm{Ag}(111)$ case to the interband transitions between the highest occupied bulklike QWS and the surface-derived $\mathrm{SS}^{+}$and $\mathrm{SS}^{-}$ states. Comparing Fig. 3 and Fig. 5, one observes that the energy difference between the upper bulklike QWS and the $\mathrm{SS}^{+}$and $\mathrm{SS}^{-}$states is close to the energy of the I and II peaks at small $q$ 's. Moreover, this assignment is supported by comparing the symmetry properties in the induced density corresponding to these modes in Fig. 7 with the symmetry of the relevant quantum states. In Fig. 7 one can see how the induced density corresponding to these modes in the 8-ML case follows the wave functions of the $\mathrm{SS}^{+}$and $\mathrm{SS}^{-}$QWSs. This kind of feature can be seen as a weak peak in the 17-ML $\mathrm{Ag}(111)$ films. In the case of $m=1 \mathrm{Ag}(111)$, these peaks are notably less pronounced, they are broader and slightly shifted to upper energies at finite momenta (see Fig. 9 for the 8-ML film). This can be explained by a very different energy dispersion of the QWS bands in $m=1 \mathrm{Ag}(111)$ slab calculations in comparison with the $m \neq 1 \mathrm{Ag}(111)$ case. Again, no such kind of peak is presented in the excitation spectra of Fig. 6 for the jellium calculations because of the lack of surface-state-like QWSs. In such calculations, some weak features due to interband transitions between QWSs can be detected only like that at $\omega \approx 1.2 \mathrm{eV}$ in Fig. 9 for the 8-ML jellium film.

\section{SUMMARY AND CONCLUSIONS}

Low-energy collective electronic excitations in thin metallic films have been investigated within linear response theory.
The self-consistent calculations were performed, taking as an example the $\operatorname{Ag}(111)$ slabs, characterized by the realistic $s-p$ electronic structure, with the thickness ranging from 1 to 10 atomic layers. We have demonstrated how quantization of electronic states in such thin films is reflected in the excitation spectra in a low-energy domain. Thus, the presence of surface-state-like electronic states in $\mathrm{Ag}(111)$ films produces a strong acoustic plasmon that converges to an acoustic surface plasmon in thicker films. Moreover, two slab-split surface states generate an interband ISP mode due to interband transitions between these states. The energy of this mode decreases in concert with reduction of the energy splitting between the surfacelike QWSs. It was found also that the interband transitions between the highest partly occupied bulklike QWS and two slab-split surface states produce notable peaks, corresponding to modes denoted as I and II, in the surface excitation spectra of $\operatorname{Ag}(111)$ thin films. All these modes can be directly probed in energy-loss experiments. The acoustic plasmons may be relevant in the interpretation of photoemission spectra as was recently demonstrated for the $\mathrm{K} / \mathrm{Be}(0001)$ system. $^{74}$ It also would be interesting to investigate the influence of the ISP and I and II modes on the optical properties of thin films. Additionally, the lateral confinement (for instance, by formation of islands ${ }^{75,76}$ ) of the strongly dispersing acoustic-like modes might have a strong effect on the light absorption in infrared range, as was recently suggested in the case of metallic nanoparticles ${ }^{77}$ and demonstrated in the case of Ag bilayer nanodisks ${ }^{58}$ and In atomic chains. ${ }^{75}$ Varying slab thickness, material, and lateral size would introduce great possibilities in the ability to tune the plasmonic properties of such atomic-scale structures.

\section{ACKNOWLEDGMENTS}

We acknowledge partial support from the University of the Basque Country (Grant No. IT-366-07), the Departamento de Educación del Gobierno Vasco, and the Spanish Ministerio de Ciencia e Innovación (Grant No. FIS201019609-C02-01). We also acknowledge the Japan Science and Technology Agency and grants-in-aid for scientific research from MEXT.
${ }^{1}$ K. Okamoto, I. Niki, A. Shvartser, Y. Narukawa, T. Mukai, and A. Scherer, Nat. Mater. 3, 601 (2004).

${ }^{2}$ K. Nakamura, K. Tanabe, and H. Atwater, Appl. Phys. Lett. 93, 121904 (2008).

${ }^{3}$ R. F. Oulton, V. J. Sorger, T. Zentgraf, R.-M. Ma, C. Gladden, L. Dai, G. Bartal, and X. Zhang, Nature (London) 461, 629 (2009).

${ }^{4}$ M. Ozaki, J.-I. Kato, and S. Kawata, Science 332, 218 (2011).

${ }^{5}$ G. Mie, Ann. Phys. 25, 377 (1908).

${ }^{6}$ F. J. García de Abajo, Rev. Mod. Phys. 79, 1267 (2007).

${ }^{7}$ N. Halas, Nano Lett. 10, 3816 (2010).

${ }^{8}$ R. H. Ritchie, Phys. Rev. 106, 874 (1957).

${ }^{9}$ T. Nagao (in U. Bovensiepen, H. Petek, M. Wolf (eds.), Dynamics at Solid State Surfaces and Interfaces, Vol. I (Wiley VCH, Manheim, 2010), p. 189.
${ }^{10}$ Z. Yaun, Y. Jiang, Y. Gao, M. Käll, and S. Gao, Phys. Rev. B 83, 165452 (2011).

${ }^{11}$ M. Rocca, Surf. Sci. Rep. 22, 1 (1995).

${ }^{12}$ A. Liebsch, Electronic Excitations at Metal Surfaces (Plenum Press, New York, 1997).

${ }^{13}$ B. Sernelius, Surface Modes in Physics (WILEY-VCH, Berlin, 2001).

${ }^{14}$ N. W. Ashcroft and N. D. Mermin, Solid State Physics (Holt, Rineheart \& Winston, New York, 1976).

${ }^{15}$ J. J. Paggel, T. Miller, and T.-C. Chiang, Science 283, 1709 (1999).

${ }^{16}$ T.-C. Chiang, Surf. Sci. Rep. 39, 181 (2000).

${ }^{17}$ M. Milun, P. Pervan, and D. P. Woodruff, Rep. Prog. Phys. 65, 99 (2002).

${ }^{18}$ F. Schiller, A. Leonardo, E. V. Chulkov, P. M. Echenique, and J. E. Ortega, Phys. Rev. B 79, 033410 (2009). 
${ }^{19}$ F. Stern, Phys. Rev. Lett. 18, 546 (1967).

${ }^{20}$ T. Nagao, T. Hildebrandt, M. Henzler, and S. Hasegawa, Phys. Rev. Lett. 86, 5747 (2001).

${ }^{21}$ D. A. Dahl and L. J. Sham, Phys. Rev. B 16, 651 (1977).

${ }^{22}$ T. Ando, A. B. Fowler, and F. Stern, Rev. Mod. Phys. 54, 437 (1982).

${ }^{23}$ J. K. Jain and S. Das Sarma, Phys. Rev. B 36, 5949 (1987).

${ }^{24}$ J. B. Williams, M. S. Sherwin, K. D. Maranowski, and A. C. Gossard, Phys. Rev. Lett. 87, 037401 (2001).

${ }^{25}$ C. A. Ullrich and G. Vignale, Phys. Rev. Lett. 87, 037402 (2001).

${ }^{26}$ G. Gumbs, Phys. Rev. B 39, 5186 (1989).

${ }^{27}$ N. D. Lang and W. Kohn, Phys. Rev. B 1, 4555 (1970).

${ }^{28}$ I. Tamm, Z. Phys. 76, 849 (1932).

${ }^{29}$ W. Shokley, Phys. Rev. 56, 317 (1939).

${ }^{30}$ S. G. Davidson and M. Stęślicka, Basic Theory of Surface States (Oxford University Press, Oxford, UK, 1992).

${ }^{31}$ V. M. Silkin, A. García-Lekue, J. M. Pitarke, E. V. Chulkov, E. Zaremba, and P. M. Echenique, Europhys. Lett. 66, 260 (2004).

${ }^{32}$ V. M. Silkin, J. M. Pitarke, E. V. Chulkov, and P. M. Echenique, Phys. Rev. B 72, 115435 (2005).

${ }^{33}$ The energies of this mode depends on the magnitude of a twodimensional (2D) in-plane momentum, $q$, and a slab thickness $d$ like $^{8} \omega_{\text {sp }}^{-}(q, d)=\frac{\omega_{p}}{\sqrt{2}}\left(1-e^{-q d}\right)^{1 / 2}$, where $\omega_{p}$ is a bulk plasma frequency.

${ }^{34}$ We define $\mathbf{q}$ as a $2 \mathrm{D}$ vector in a plane parallel to a slab. Note that, due to the isotropic symmetry of slabs along its surface presumed in the present paper, all the quantities depend on the magnitude of the $2 \mathrm{D} \mathbf{q}$ vector only.

${ }^{35}$ B. N. J. Persson and E. Zaremba, Phys. Rev. B 31, 1863 (1985).

${ }^{36}$ K.-D. Tsuei, E. W. Plummer, A. Liebsch, E. Pehlke, K. Kempa, and P. Bakshi, Surf. Sci. 247, 302 (1991).

${ }^{37}$ H. Ishida and A. Liebsch, Phys. Rev. 54, 14127 (1996).

${ }^{38}$ V. M. Silkin, E. V. Chulkov, and P. M. Echenique, Phys. Rev. Lett. 93, 176801 (2004).

${ }^{39}$ F. Aryasetiawan and K. Karlsson, Phys. Rev. Lett. 73, 1679 (1994).

${ }^{40}$ E. E. Krasovskii and W. Schattke, Phys. Rev. B 59, R15609 (1999).

${ }^{41}$ A. G. Eguiluz, Phys. Rev. Lett. 51, 1907 (1983).

${ }^{42}$ A. G. Eguiluz, Phys. Rev. B 31, 3303 (1985).

${ }^{43}$ E. V. Chulkov, V. M. Silkin, and P. M. Echenique, Surf. Sci. 391, L1217 (1997).

${ }^{44}$ E. V. Chulkov, V. M. Silkin, and P. M. Echenique, Surf. Sci. 437, 330 (1999).

${ }^{45}$ K. Pohl, B. Diaconescu, G. Vercelli, L. Vattuone, V. M. Silkin, E. V. Chulkov, P. M. Echenique, and M. Rocca, Europhys. Lett. 90, 57006 (2010).

${ }^{46}$ E. E. Krasovskii, V. M. Silkin, V. U. Nazarov, P. M. Echenique, and E. V. Chulkov, Phys. Rev. B 82, 125102 (2010).

${ }^{47}$ I. Matsuda, T. Tanikawa, S. Hasegawa, H. W. Yeom, K. Tono, and T. Ohta, J. Surf. Sci. Nanotech. 2 , 169 (2004).

${ }^{48}$ N. J. Speer, S. J. Tang, T. Miller, and T.-C. Chiang, Science 314, 804 (2006).
${ }^{49}$ T. Nagao, T. Hildebrandt, M. Henzler, and S. Hasegawa, Surf. Sci. 493, 680 (2001).

${ }^{50} \mathrm{~T}$. Nagao, OYO BUTURI 73, 1312 (2004), and references therein (in Japanese).

${ }^{51}$ T. Nagao, S. Yaginuma, T. Inaoka, V. U. Nazarov, T. Nakayama, and M. Aono, Proc. SPIE 6641, 664116 (2007).

${ }^{52}$ Y. Liu and R. F. Willis, Surf. Sci. 603, 2115 (2009).

${ }^{53}$ E. P. Rugeramigabo, T. Nagao, and H. Pfnür, Phys. Rev. B 78, 155402 (2008).

${ }^{54}$ T. Langer, J. Baringhaus, H. Pfnür, H. W. Schumacher, and C. Tegenkamp, New J. Phys. 12, 033017 (2010).

${ }^{55}$ Y. Liu, R. F. Willis, K. V. Emtsev, and Th. Seyller, Phys. Rev. B 78, 201403(R) (2008).

${ }^{56}$ A. Politano, V. Formoso, E. Colavita, and G. Chiarello, Phys. Rev. B 79, 045426 (2009).

${ }^{57}$ A. Politano and G. Chiarello, Surf. Rev. Lett. 16, 171 (2009).

${ }^{58}$ H. J. Qin, Y. Gao, J. Teng, H. X. Xu, K. H. Ku, and S. W. Gao, Nano Lett. 10, 2961 (2010).

${ }^{59}$ L. Marušić, V. Despoja, and M. S̆unjić, J. Phys. Condens. Matter 18, 4253 (2006).

${ }^{60}$ Z. Yuan and S. W. Gao, Surf. Sci. 602, 460 (2008).

${ }^{61}$ V. U. Nazarov, S. Nishigaki, and T. Nagao, Phys. Rev. B 66, 092301 (2002).

${ }^{62}$ T. Inaoka, T. Nagao, S. Hasegawa, T. Hildebrandt, and M. Henzler, Phys. Rev. B 66, 245320 (2002).

${ }^{63}$ A. Bergara, V. M. Silkin, E. V. Chulkov, and P. M. Echenique, Phys. Rev. B 67, 245402 (2003).

${ }^{64}$ T. Inaoka, Phys. Rev. B 71, 115305 (2005).

${ }^{65}$ A. Rodriguez-Prieto, V. M. Silkin, A. Bergara, and P. M. Echenique, Surf. Sci. B 600, 3856 (2006).

${ }^{66}$ M. Pletyukhov and V. Gritsev, Phys. Rev. B 74, 045307 (2006).

${ }^{67}$ Z. Yuan and S. W. Gao, Comp. Phys. Commun. 180, 466 (2009).

${ }^{68}$ D. Pines, Can. J. Phys. 34, 1379 (1956).

${ }^{69}$ B. T. Geilikman, Sov. Phys. JETP 21, 796 (1965).

${ }^{70}$ R. F. Wood, B. E. Sernelius, and A. L. Chernyshev, Phys. Rev. B 66, 014513 (2002).

${ }^{71}$ J. M. Pitarke, V. U. Nazarov, V. M. Silkin, E. V. Chulkov, E. Zaremba, and P. M. Echenique, Phys. Rev. B 70, 205403 (2004).

${ }^{72}$ B. Diaconescu, K. Pohl, L. Vattuone, L. Savio, Ph. Hofmann, V. M. Silkin, J. M. Pitarke, E. V. Chulkov, P. M. Echenique, D. Farías, and M. Rocca, Nature (London) 448, 57 (2007).

${ }^{73}$ S. J. Park and R. E. Palmer, Phys. Rev. Lett. 105, 016801 (2010).

${ }^{74}$ V. M. Silkin, B. Hellsing, L. Walldén, P. M. Echenique, and E. V. Chulkov, Phys. Rev. B 81, 113406 (2010).

${ }^{75}$ T. Nagao et al., Sci. Technol. Adv. Mater. 11, 054506 (2010).

${ }^{76}$ J. M. Pitarke, V. M. Silkin, E. V. Chulkov, and P. M. Echenique, Rep. Prog. Phys. 70, 1 (2007).

${ }^{77}$ A. Traverse, T. Girardeau, C. Prieto, D. D. S. Meneses, and D. Zanghi, Europhys. Lett. 81, 47001 (2008). 